Recepción: 15 / 04 / 2017

Ciencias Médicas

Aceptación: 01 / 05 / 2017

Articulo Científico

Publicación: 15 / 05 / 2017

\title{
Evaluación del dolor torácico como predictor de síndrome metabólico en pacientes de emergencia hospitalaria
}

\begin{abstract}
Evaluation of thoracic pain as a predictor of metabolic syndrome in hospital emergency patients
\end{abstract}

Avaliação de dor no peito como um preditor de síndrome metabólica em pacientes de emergência do hospital

\author{
Cleotilde F. López-Loor \\ dra.fabianalopezloor@yahoo.com \\ Maria L. Acuña-Cumba " \\ draluisaacunacumba@gmail.com
}

Martha B. Baquerizo-Cabrera ${ }^{\text {"m }}$ martha.baquerizoc@ug.edu.ec

Correspondencia: dra.fabianalopezloor@yahoo.com

\footnotetext{
Magister en Emergencias Médicas; Medico; Universidad de Guayaquil, Ecuador.

Magister en Bioquímica Clínica; Diplomado en Docencia Superior; Especialista en Patología Clínica; Doctora Medicina y Cirugía; Universidad de Guayaquil, Ecuador.

III. Magister en Gerencia Educativa; Especialista en Gestión de Procesos Educativos; Diplomado en Docencia Superior; Doctora en Medicina y Cirugía; Universidad de Guayaquil, Ecuador.
} 
Cleotilde F. López-Loor; Maria L. Acuña-Cumba; Martha B. Baquerizo-Cabrera

\section{Resumen}

Los pacientes que acuden al servicio de emergencia, una gran mayoría es con dolor torácico agudo, siendo una patología prevalente en la actualidad y un detonante para el un aumento del riesgo cardiovascular, en relación con el síndrome metabólico. Se realizó una investigación cuantitativa, analítica retrospectiva, transversal no experimental con el objetivo de determinar la importancia del dolor torácico agudo en relación con el síndrome Metabólico como factor predictor, que ingresan al área de emergencia del Hospital Velasco Ibarra en el año 2015. Resultados: De los 534 casos atendidos con dolor torácico agudo se analizaron 30 casos que presentaron relación con síndrome coronario agudo y síndrome metabólico, de los cuales como factores de riesgo predominó la diabetes mellitus con el $86,6 \%$ seguido de la hipertensión arterial que predominó con el $80 \%$; el $76,67 \%$ correspondió a pacientes con trigliceridemia, el $66,67 \%$ con obesidad y el $60 \%$ con colesterol, en cuanto al grupo etáreo se identificó que los mayores de 60 años y el género masculino tuvieron el $86,67 \%$ del universo sobre 30 pacientes. Conclusión: de los 534 pacientes que acudieron al servicio de emergencia 5,61\% presentaron dolor torácico agudo relacionado con el síndrome coronario agudo y síndrome metabólico que equivale a 30 casos por lo que refleja que el personal de emergencias no está capacitado para el diagnóstico de esta patología y siendo una morbilidad prevalente en la actualidad, en esta investigación se definirá un plan de estrategia para esta unidad hospitalaria y disminuir la morbimortalidad de dicha población.

Palabras clave: Dolor torácico agudo; síndrome metabólico; síndrome coronario agudo y factores de riesgo. 


\begin{abstract}
Patients who come to the emergency department, a large majority are with acute chest pain, being a prevalent pathology currently and a trigger for an increased cardiovascular risk, in relation to the metabolic syndrome. A quantitative, retrospective, non-experimental cross-sectional study was carried out to determine the importance of acute chest pain in relation to the Metabolic syndrome as a predictor, which entered the emergency area of Velasco Ibarra Hospital in 2015. Results: Of the 534 cases treated with acute chest pain, 30 cases with acute coronary syndrome and metabolic syndrome were analyzed. Of these, diabetes mellitus predominated, with $86.6 \%$ followed by hypertension, which predominated with $80 \% ; 76.67 \%$ corresponded to patients with triglyceridemia, $66.67 \%$ with obesity and $60 \%$ with cholesterol. As to the age group, it was identified that those over 60 years old and the male gender had $86.67 \%$ of the universe Over 30 patients. Conclusion: Of the 534 patients who attended the emergency service, $5.61 \%$ presented acute chest pain related to acute coronary syndrome and metabolic syndrome, which corresponds to 30 cases, thus reflecting that emergency personnel are not qualified for the diagnosis of This pathology and being a morbidity prevalent at present, in this research will define a strategy plan for this hospital unit and decrease the morbidity and mortality of said population.
\end{abstract}

Key words: Acute thoracic pain, metabolic syndrome, acute coronary syndrome and risk factors. 


\section{Resumo}

Os pacientes que vêm ao serviço de emergência, a grande maioria são com dor torácica aguda, sendo uma patologia prevalente atualmente e um gatilho para um aumento do risco cardiovascular, em relação à síndrome metabólica. Realizou-se um estudo transversal quantitativo, retrospectivo e não-experimental para determinar a importância da dor torácica aguda em relação à síndrome metabólica como preditor, que entrou na área de emergência do Hospital Velasco Ibarra em 2015. Resultados: Dos 534 Casos tratados com dor torácica aguda, 30 casos com síndrome coronariana aguda e síndrome metabólica foram analisados. Destes, predominou o diabetes mellitus, com 86,6\% seguido de hipertensão, que predominou com $80 \% ; 76,67 \%$ corresponderam a pacientes com trigliceridemia, 66,67\% com obesidade e $60 \%$ com colesterol. Quanto ao grupo etário, foi identificado que aqueles com mais de 60 anos eo sexo masculino tinham 86,67\% do universo. Mais de 30 pacientes. Conclusão: Dos 534 pacientes atendidos no serviço de emergência, 5,61\% apresentaram dor torácica aguda relacionada à síndrome coronariana aguda e à síndrome metabólica, o que corresponde a 30 casos, refletindo que o pessoal de emergência não está qualificado para o diagnóstico desta patologia e Morbidade prevalente no presente, nesta pesquisa irá definir um plano de estratégia para esta unidade hospitalar e diminuir a morbidade e mortalidade da referida população.

Palavras chave: dor torácica aguda; síndrome metabólico; síndrome e fatores de risco coronariano agudo. 


\section{Introducción.}

Los pacientes con dolor torácico agudo conforman una proporción muy importante de todas las urgencias y hospitalizaciones médicas por esa razón la identificación de los pacientes con síndrome coronario agudo supone un reto diagnóstico sobre todo cuando los síntomas son dudosos o no hay pruebas electrocardiográficas; la determinación del síndrome coronario agudo se basa en la combinación de la presentación clínica (DT de tipo no traumático sugestivo que tiene un origen isquémico),variaciones electrocardiográficas y el acrecentamiento de la concentración de enzimas miocárdicas, el DT sugiere un comienzo de tipo isquémico el cual es el detonante para el proceso de diagnóstico y, junto con las alteraciones del electrocardiograma.

El problema identificado se observa en el área de emergencia de entidades de salud en pacientes que llegan con dolor torácico agudo, quienes deben ser hospitalizados con un diagnostico primario de síndrome coronario agudo sin elevación del segmento " $\mathrm{T}$ " siendo este el proceso de mayor interés en las áreas de urgencias en su fase pre-hospitalaria y hospitalaria, tanto por la necesidad de actuación terapéutica inmediata como por la necesidad de realizar el diagnóstico diferencial, esta enfermedad se presenta en mayor forma en los países desarrollados-

Por lo tanto, la pregunta de investigación sería ¿Es el dolor torácico agudo un determinante asociado al síndrome coronario agudo en los pacientes que llegan al servicio de emergencia en el Hospital Velasco Ibarra durante el año 2015?

Existe una alta incidencia de casos de dolor torácico agudo en el área de emergencia, lo que requiere un diagnóstico precoz analizando una problemática preocupante que genera elevada morbimortalidad, gastos de salud y consecuencias sociales y económicas para el propio paciente y su familia. El objeto de estudio es la relación del dolor torácico con el síndrome coronario agudo y 
el síndrome metabólico. Campo de acción pacientes con dolor torácico agudo que acuden al área de emergencia del Hospital.

El presente estudio tiene como objetivo determinar el dolor torácico agudo con el síndrome metabólico en pacientes de emergencia del hospital José María Velasco Ibarra de Tena. Los objetivos específicos Relacionar el dolor torácico agudo según género y grupo etáreo. Determinar la relación del dolor torácico agudo con el síndrome coronario y metabólico. Diseñar un plan de estrategia identificando las actividades para incidir en el estilo de vida de esa población previniendo el síndrome metabólico predisponente de las enfermedades coronarias.

\section{Materiales y métodos.}

Es un estudio de tipo teórico y empírico, el primero consiste en "Permiten descubrir en el objeto de investigación las relaciones esenciales y las cualidades fundamentales, no detectables de manera sensoperceptua y por ello se apoya básicamente en los procesos de abstracción, análisis, síntesis, inducción y deducción.", y el estudio empírico refiere "Su aporte al proceso de investigación es resultado fundamentalmente de la experiencia. Estos métodos posibilitan revelar las relaciones esenciales y las características fundamentales del objeto de estudio, accesibles a la detección sensoperceptual, a través de procedimientos prácticos con el objeto y diversos medios de estudio" se tomara con enfoque transversal y analítico porque consiste "Estudian las variables de forma simultánea en un momento dado" (Vásquez 2011), y no experimental ya que se realizó sin manipular las variables deliberadamente. Basándonos tan solo en la observación de fenómenos dentro de su contexto para después analizarlos por lo que se recogió la información de los 30 pacientes atendidos durante el período de estudio en el en área de emergencia del Hospital Velasco Ibarra. 
Métodos

Para el presente trabajo se empleó el método cuantitativo, retrospectivo, transversal no experimental; este estudio se inicia a partir de enero a diciembre del año 2015 para lo cual se realizó la recolección de datos de las historias clínicas de los pacientes atendidos en el área de emergencia de Hospital Velasco Ibarra con síntomas de dolor torácico agudo asociado al síndrome coronario agudo para sus posteriores procesamientos. Para el análisis y síntesis de la información se realizó en hoja de cálculo Excel y para el análisis se utilizó el programa SPSS, para riesgo relativo, niveles de confianza y porcentajes los cuales se presentarán en cuadros que generan los resultados de la investigación.

El universo fueron los 534 pacientes atendidos en el área de emergencia con dolor torácico agudo del Hospital Velasco Ibarra de enero a diciembre del año 2015 y la muestra los 30 pacientes con diagnóstico de dolor torácico agudo asociado a síndrome coronario agudo y relacionado con Síndrome metabólico.

\section{Resultados.}

Antecedentes de la unidad de análisis o población

El Hospital fue fundado en 1955 por la Asistencia Social y Entregado a la Misión Josefina de Napo, siendo su Administrador el Obispo Maximiliano Spiller, con una dotación de 40 camas, el mismo funcionó en el local situado frente al Parque Central de Tena. En 1973, el Señor Contralor General de la Nación mediante telegrama, nombra a la Sra. Inés Espinosa de Espinosa para que se realice la entrega recepción de la casa de salud, fecha desde la cual toma a cargo la Dirección Provincial de Salud de Napo. En 1986, se inaugura el nuevo edificio situado en la Av. 15 de 
noviembre y Eloy Alfaro, con dotación normal de 70 camas, incrementándose personal en todas sus áreas. En la actualidad el Hospital José María Velasco Ibarra, durante sus veinte y nueve años de servir a la comunidad en la planta física en la que se encuentra al momento, ha sido un referente de salud de la provincia y de la región Amazónica. El hospital José María Velasco Ibarra pertenece al Ministerio de Salud Publica ubicado en la ciudad de Tena, una población de 4500 habitantes, capital de la provincia del Napo ubicado en la región amazónica, localizada a $120 \mathrm{~km}$ al sudeste de la ciudad de Quito e inicio sus operaciones en el año 1986 y cuenta con una capacidad de 120 camas.

Los pacientes que acuden al hospital José María Velasco Ibarra al servicio de urgencias con dolor torácico suponen una proporción significativa del volumen de urgencias y los ingresos hospitalarios por dolor torácico finalmente se demuestra que no tenían un síndrome coronario agudo. El área de Cardiología y la Unidad Coronarias propone un protocolo de funcionamiento de las unidades de dolor torácico, bien sean funcionales o físicas, ubicadas en el área de urgencias, atendidas por personal especializado y dirigidas por un cardiólogo. Todo paciente con diagnóstico de SICA definitiva o altamente probable, debe ser admitido de inmediato a una unidad de cuidados coronarios o de terapia intensiva con facilidades de atención coronaria y preferentemente que cuenten con posibilidades de reperfusión farmacológica o intervensionista.

\section{Diagnóstico o estudio de campo}

El grupo etáreo refiere al tiempo que una persona ha vivido a partir del nacimiento 0-19 años, 19 a 39 años, 39 a 59 años y más de 60 años y se conoce que cuatro de cinco pacientes que fallecen por enfermedad cardiovascular son mayores de 65 años. Los géneros masculinos tienen mayor riesgo de enfermedad cardiovascular que las mujeres, y son afectados en edades más tempranas, luego de la menopausia el riesgo de las mujeres aumenta, pero aún es menor que en el 
sexo masculino. La Glicemia en rangos normal en la sangre se encuentra entre $70 \mathrm{mg} / \mathrm{dl}$ y 100 $\mathrm{mg} / \mathrm{dl}$ en ayunas y cuando se incrementa se denomina Diabetes mellitus, esta es un riesgo de desarrollar enfermedad cardiovascular. Más del $80 \%$ de los pacientes diabéticos pueden padecer alguna afección de este tipo. (Loàisiga Ruiz, 2016)

Las Anormalidades en el ECG se refiere a la correlación entre el registro electrocardiográfico con la actividad eléctrica normal del corazón. En el ECG normal nos encontramos con una primera onda, Onda $\mathrm{P}$, que corresponde a la despolarización de ambas aurículas, derecha e izquierda superpuestas. El estímulo se frena en el nodo AV, por lo que durante este tiempo no se registra actividad eléctrica, para inmediatamente iniciar la despolarización ventricular, dando lugar al complejo QRS, que se sigue de otro período isoeléctrico, para finalizar con la onda $\mathrm{T}$ de repolarización ventricular. (Carmona 2011)

El Lugar de residencia es donde vive el caso de estudio al momento de la recolección de datos y se midió de la siguiente manera si pertenece a la Zona urbana: Se encuentra en la jurisdicción del Cantón de Tena y cuenta con los servicios básicos de agua, desagüe y energía eléctrica; la zona periurbana la cual se sitúan a los alrededores de la jurisdicción y cuenta con dos servicios básicos agua y energía eléctrica Zona rural que carece de servicios básicos.

El dolor torácico agudo se denomina a la sensación álgida localizada en la zona situada entre el diafragma y la fosa supraclavicular:

El tipo opresivo de intensidad variable localizado en región precordial, con irradiación a cuello, hombro y brazo izquierdo; acompañado de síntomas neurovegetativos, aparece en actividad, y se alivia con el reposo y/o nitratos. Los factores de riesgo cardiovascular son medianamente predictores de la probabilidad de enfermedad coronaria en pacientes asintomáticos y sintomáticos; 
los estilos de vida, el hábito de fumar menor disponibilidad para preocuparse por su salud, minimización de síntomas etc.(Mosca L, 2010) entre ellos el sobrepeso predispone a desarrollar enfermedades cardiovasculares Es perjudicial porque incrementa el esfuerzo a que es sometido el corazón, y se vincula a la enfermedad coronaria por su influencia negativa sobre el colesterol y la diabetes tipo II. (Loàisiga Ruiz, 2014)

\section{Discusión.}

\section{Contrastación Empírica}

Se observa que la mayoría de casos de pacientes con evolución clínica pertenece al grupo etáreo de más de 60 años con mayor atención en comparación con el estudio de Castro en Toluca Estado de México sobre "Prevalencia de síndrome coronario agudo tipo infarto agudo de miocardio con elevación del segmento ST en el servicio de urgencias en el año 2013” identifica 53 casos fueron por presentar dolor torácico compartible con síndrome coronario agudo se aprecian cambios respecto al grupo etario siendo más frecuentes en nuestra población entre los 35-45 años de edad respecto a la media nacional de 52 años, por otra parte los factores de riesgo cardiovascular permanecen con una tendencia similar a las nacionales con una marcada tendencia a la relación diabetes.

Así mismo se observa que la mayoría de los casos presentados de dolor torácico fue en el género masculino como también observamos en el estudio realizado en Ambato - Ecuador por Vásquez denominado "El Síndrome Metabólico como factor de riesgo en pacientes admitidos con Síndrome Coronario agudo en el servicio de cardiología del Hospital Provincial Docente Ambato" describe que se determinó que en la población estudiada el riesgo mayor esta en los pacientes con 
síndrome metabólico de sexo masculino para presentar Síndrome coronario de alto riesgo con un RR. 1,7 en relación a RR. 0 en la población femenina.

Así mismo se observa que la mayoría de pacientes que son ingresados con dolor torácico son de procedencia urbana en comparación con el estudio realizado por Madrid Miller en el año 2010 en México "Síndrome metabólico, impacto clínico y angiográfco en pacientes con síndrome coronario agudo" refiere como resultados de 971 pacientes con SICA, 421 (43.4 \%) tuvieron síndrome metabólico. La edad fue de $63.37 \pm 9.95$ años versus $61.77 \pm 12.2$ años; $66.7 \%$ versus $72.8 \%$ hombres; $70.3 \%$ versus $33.3 \%$ diabéticos; $87.6 \%$ versus $40.5 \%$ hipertensos y dislipidemia $70.5 \%$ versus $26.9 \%$, respectivamente para los grupos A y B. El diagnóstico de SICA con elevación del ST fue más frecuente en el grupo A, así como mayor severidad en el grado de enfermedad arterial coronaria y mayor incidencia de eventos cardiovasculares mayores (Madrid, 2010).

El factores de riesgo con un 86,67\% /(26) casos corresponden a la diabetes mellitus tipo II como se observa en el estudio "Morbimortalidad y consumo de recursos asociados tras síndrome coronario agudo en una población española” realizado en España por Sicras-Mainar en el año 2011 donde el análisis estadístico: regresión logística, curvas Kaplan-Meier y ANCOVA; p < 0,05 teniendo como resultados que fueron incluidos 1.020 pacientes. Edad media: 69 años; 65\% hombres. Grupos: SCA con elevación ST ( $\mathrm{N}=632 ; 62 \%)$. Comorbilidades: hipertensión (56\%), dislipemia (46\%) y diabetes (38\%). Prevalencia de SM: 59\% (IC 95\%: 56-62\%) (Sicras-Mainar, 2011).

La mayor parte de las atenciones fueron realizadas en el mes de agosto con siete casos de atención con diagnóstico de dolor torácico y según Bodi en el estudio "Pronóstico a corto plazo de los pacientes ingresados por probable síndrome coronario agudo sin elevación del segmento ST. 
Papel de los nuevos marcadores de daño miocárdico y de los reactantes de fase aguda" realizado en el España refiere la puntuación del dolor torácico fue 10,2 $\pm 2,8$. En el ECG se detectó descenso del ST en el 23\% de los casos y de la onda T en el 10\%. Un 54\% de los casos presentó elevación de la troponina I y un $20 \%$ presentó simultáneamente cambios electrocardiográficos y elevación de la troponina I.

Refiere que la mayoría tienen como antecedentes un familiar con patologías cardiovasculares así mismo según Reina Toral en el estudio realizado en España en el año 2014 "Diferencias en los resultados de la atención a los pacientes con síndrome coronario agudo con elevación del segmento ST (SCACEST) en función del acceso inicial a hospitales con o sin sala de hemodinámica en Andalucía" refiere que realizaron un estudio de cohortes retrospectivo sobre los 11.122 pacientes con SCACEST, del total de pacientes analizados, 5.728 (51,5\%) fueron atendidos en hospitales con SH, frente a $5.394(48,5 \%)$ en hospitales sin SH. De los pacientes atendidos en hospitales con SH, a $1.891(33,0 \%)$ se les realizó algún tipo de intervencionismo coronario percutáneo (ICP), y a 2.052 $(35,8 \%)$ tratamiento fribrinolítico, mientras que en hospitales sin SH, se realizó ICP a 349 (6,5\%) pacientes y fibrinolisis a $2.665(44,8 \%)(\mathrm{p}<0,001)($ Reina Toral 2014).

Además se observa que son los pacientes de género masculino quienes controlan sus valores de glicemia y en el estudio de Ortega y Piedra realizado en Quito - Ecuador denominado "Hiperglicemia y volumen plaquetario medio como predictores tempranos del síndrome coronario agudo tipo iam sest, en pacientes con dolor torácico atendidos en el departamento de emergencia del hospital "Carlos Andrade Marín" donde se observaron los resultados de los 133 pacientes incluidos en el trabajo, para la hiperglicemia >140 mg/dl (AUC: 0,923; IC 95\%: 0,879-0,967; p<0,05) la sensibilidad fue del 71,9\%, especificidad de 90,1\%, valor predictivo positivo $69,7 \%$, valor 
predictivo negativo 91\%, razón de verosimilitud positivo 7,259 y razón de verosimilitud negativo 0,312 (Ortega y Piedra, 2015).

Se observa que los pacientes atendidos con control de su tensión arterial la mayoría son de género masculino y en el estudio "Morbimortalidad y consumo de recursos asociados tras síndrome coronario agudo en una población española” realizado en España por Sicras-Mainar en el año 2011 determinaron en un estudio multicéntrico y retrospectivo basado en revisión de registros médicos, de dos años de seguimiento con pacientes de 6 centros de Atención Primaria y dos hospitales. Grupos: SCA con elevación ST (N = 632; 62\%). Comorbilidades: hipertensión (56\%), dislipemia (46\%) y diabetes (38\%). Prevalencia de SM: 59\% (IC 95\%: 56-62\%) (Sicras-Mainar, 2011).

Los niveles de colesterol elevados correspondieron a la población masculina y comparando el estudio realizado en Colombia del autor Chavarriaga en el año 2014 denominado "Características epidemiológicas, clínicas, tratamiento y pronóstico de los pacientes con diagnóstico de síndrome coronario agudo en unidad especializada" donde realizan estudio epidemiológico, observacional, descriptivo, longitudinal, retrospectivo tipo serie de casos. Como resultados: un total de 154 pacientes fueron analizados, 30\% $(\mathrm{n}=47)$ por angina inestable, 37\% y $(\mathrm{n}=56)$ por IAMST y $33 \%$ $(n=51)$ por IAMNST. El promedio de edad fue de $62 \pm 13$ años, hombres $54 \%(n=83)$. Los factores de riesgo asociados fueron: hipertensión arterial $66 \%(\mathrm{n}=101)$, dislipidemia $23 \%(\mathrm{n}=35)$, diabetes mellitus $18 \%(\mathrm{n}=27)$, obesidad 5,1\% $(\mathrm{n}=8)$ y tabaquismo 52\% $(\mathrm{n}=80)($ Chavarriaga, 2011)..

En los pacientes con circunferencia Abdominal en valores patológicos la mayor parte fue para el género masculino y según el estudio de Cárdenas -Villarreal en el año 2012 en México “Identificación de componentes del síndrome metabólico en pacientes mexicanos hospitalizados por síndrome isquémico coronario agudo: una herramienta para la prevención” en el cual Participaron 
65 pacientes que ingresaron a la unidad de cuidados intensivos coronarios con infarto agudo del miocardio o angina inestable. Se diagnosticó SM en base en los criterios del Adult Treatment Pannel (ATP-III); siendo los resultados que la prevalencia total de SM fue 84,6\% (IC 95\%:75,6 a 93,6), se presentó más en mujeres, en personas con obesidad según índice de masa corporal (IMC) y con antecedentes familiares de diabetes y dislipidemia. (Cárdenas -Villarreal, 2012)

\section{Conclusiones.}

Con el presente estudio se puede concluir que de los 534 pacientes que acudieron al servicio de emergencia $5,61 \%$ presentaron dolor torácico agudo relacionado con el síndrome coronario agudo y síndrome metabólico que equivale a 30 casos por lo que refleja que el personal de emergencias no está capacitado para el diagnóstico de esta patología y siendo una patología prevalente en la actualidad, en esta investigación se definirá un plan de estrategia para esta unidad hospitalaria y disminuir la morbimortalidad de dicha población. La preexistencia en la prevalencia de factores de riesgo entre los pacientes ingresados con dolor torácico agudo como síntomas predictores de síndrome coronario agudo y las estadísticas internacionales sobre los factores de riesgo cardiovascular. Fundamentalmente en nuestro país debe realizarse énfasis en programas de prevención y control de hipertensión arterial, diabetes y de la obesidad, teniendo un alto porcentaje de frecuencia en pacientes de género masculino, conjuntamente en modificar estilos de vida y hábitos nocivos como el tabaquismo, el sedentarismo y la mala alimentación que suman en la alta incidencia de riesgo de infarto posteriormente revascularización coronaria y muerte.

La frecuencia del síndrome metabólico en pacientes con síndrome coronarios agudo tiene una gran incidencia y en nuestro país toma mayor predisposición de mortalidad por las comorbilidades asociando un mal pronóstico intrahospitalario. Es importante que dentro de la 
calidad de la atención de los pacientes con dolor torácico el oportuno diagnóstico permitiría detectar errores en el manejo y procurar extender la calidad de vida ya que mejora el subdiagnostico debido a que los pacientes al referir mejoría de la sintomatología no regresan a la consulta hospitalaria.

Los cuadros clínicos inciertos crean dificultad de diagnósticos definitivos para la cual se sugiere que se realice una evaluación clínica más avanzada con los exámenes para la aplicación de los algoritmos de diagnóstico y reiterados exámenes de gabinete y estos colaboren con el manejo del dolor torácico agudo. La severidad también se observa con la respuesta inflamatoria y protrombótica asociada a patologías de enfermedades arteriales coronarias y como resulta se incrementa los eventos cardiovasculares. Los factores de riesgo deben considerarse dentro de la evaluación, pronóstico y tratamiento en todos los pacientes que cursan enfermedades vasculares.

\section{Bibliografía.}

Amsterdam, E., Wenger, N., Brindis, R., Casey, D., Ganiats, T., Holmes, D.,Zieman, S. (2014). AHA/ACC guideline for the management of patients with non-ST-elevation acute coronary syndromes: a report of the American College of Cardiology/American Heart Association Task Force on Practice Guidelines. Circulation, 130(25), 344-426. Recuperado de la base de datos de PubMed

Amsterdam, EA., Wenger, NK., Brindis, RG. (2014). AHA/ACC Guideline for the Management of Patients With Non-ST-Elevation Acute Coronary Syndromes: Executive Summary: A Report of the American College of Cardiology/American Heart Association Task Force on Practice Guidelines. J Am Coll Cardiol, 64, 2645. Recuperado de la base de datos de PubMed.

American College of Endocrinology Task Force on the Insulin (2013). The metabolic syndrome and total and cardiovastion Statement on the Insulin Resistance Syndrome.. Endocr Pract; 2013.9:236-252.

American Heart Association (2010 ). Guidelines for Cardiopulmonary Resuscitation and Emergency Cardiovascular Care. Circulation. 2010;122:S640.

Bishop CR, Dargan PI, Greene SL, Garnham F, Wood DM. (2010). Emergency department presentations with suspected acute coronary syndrome - frequency of self-reported cocaine use. Eur J Emerg Med. 2010.17:164-6

Bayés de Luna A, Brugada J, Baranchuk A, et al. (2012). Current electrocardiographic criteria for diagnosis of Brugada pattern: a consensus report. J Electrocardiol. 2012; 45: 433-442 
Contreras-Leal É, Santiago-García J. (2011). Obesidad, síndrome metabólico y su impacto en las enfermedades Cardiovasculares. Rev. Biomed. [revista en Internet]. 2011 Disponible en: http:// www.medigraphic.com/pdfs/revbio/bio-2011/bio113e.pdf

European Heart Network (2010). European Cardiovascular Disease Statistics. edition. 2010.

European Heart Journal (2010). Guidelines on myocardial revascularization 31, 2501-2555

European Heart Journal (2012). Guidelines for the management of acute myocardial infarction in patients presenting with ST-segment elevation.

Hamm CW, Bassand JP, Agewall S, Bax J, Boersma E y Cols. (2011). Guidelines for the anagement of acute coronary syndromes in patients presenting without persistent ST-segment elevation: The Task Force for the management of acute coronary syndromes (ACS) in patients presenting without persistent STsegment elevation of the European Society of Cardiology (ESC). Eur Heart J. 2011; 32:2999-3054.

Kutty RS, Jones N, Moorjani N. (2013). Mechanical complications of acute myocardial infarction. Cardiol Clin. 2013 Nov;31(4):519-31.

Lopez-Sendon J, Gurfnkel EP, Lopez de Sa E, Agnelli G, Gore JM, Steg PG, et al. (2010). Factors related to heart rupture in acute coronay syndromes in the Global Registry of Acute Coronary Events. Eur Heart J. 2010 Jun;31(12):1449-56.

McManus DD, Gore AJ, Yarzebski JE, Spencer FV, Lessard DA, Goldberg RJ.(2011). Recent trends in the incidence, treatment, and outcomes of patients with STEMI and NSTEMI. Am J Med. 2011; 124:407.

Meier B, Frank B, Wahl A, Diener HC. (2012). Secondary stroke prevention: patent foramen ovale, aortic plaque, and carotid stenosis. Eur Heart J. 2012 Mar;33(6):705-13, 713a, 713b

Ministerio de Salud Pública (2012). Expediente Único para la Historia Clínica Manejo del registro Médico Orientado por Problemas Guía para el análisis Rediseño de los formularios básicos. 2011: 32: 3-5

Montefortea N, Napolitanoa C, Prioria S. (2012). Genética y arritmias: aplicaciones diagnósticas y pronósticas. Rev Esp Cardiol. 2012;65(3):278-286.

Monteiro R, Azevedo I. (2010). Chronic infammation in obesity and the Metabolic Syndrome. Mediators of Infammation [en Internet]. 2010 [Acceso 20 Mayo 2013]. Disponible en: http://www.hindawi.com/journals/mi/2010/28964

Organización Mundial de la Salud (2012). Estadísticas Sanitarias Mundiales 2012. [en Internet] Ginebra: OMS; 2012. Disponible en: http://www.who.int/gho/publications/world_health_statistics/ ES_WHS2012_Full.pdf

Postema PG, Vlaar Ap, DeVries JH, Tan HL (2011). Familial Brugada syndrome uncovered by hyperkalaemic diabetic ketoacidosis. Europace. 2011 Oct;13(10):1509-10.

Recasens L, Meroño O, Ribas N.( 2013) Hiperpotasemia simulando un síndrome de Brugada. Rev Esp Cardiol. 2013;66(4):309. 
Sanchez-Jimenez EF.(2013). Initial clinical presentation of Takotsubo cardiomyopathy with a focus on electrocardiographic changes: A literature review of cases. Wold J Cardiolog. 2013. July 26; 5(7): 228-241.

Simmons R, Alberti K, Gale E, Colagiuri S, Tuomilehto J, Qiao Q, et al. (2010) The metabolic syndrome: useful concept or clinical tool? Report of a WHO Expert Consultation. Diabetologia 2010. Disponible en: http://link.springer.com/content/ pdf/10.1007\%2Fs00125-009-1620-4.pdf

Sinay I, Costa J, de Loredo L, Ramos O, Lúquez H. da Silva Filho Rl, et al. (2010). Epidemiologia, diagnostico, control, prevención y tratamiento del síndrome metabólico en adultos. Rev. ALAD [revista en Internet]. 2010: 25-44. Disponible en: http://revistaalad.com.ar/ pdfs/100125-44.pdf

Vahanian A, Alferi O, Andreotti F, Antunes MJ, Barón-Esquivias G, Baumgartner H. (2012). Guidelines of management of valvular heart Disease. Eur Heart J 2012:33(19):2451-96.

Westaby S, Kharbanda R, Banning AP. (2011). Cardiogenic shock in ACS. Part 1: prediction, presentation and medical therapy. Nat Rev Cardiol 2011;9(3):158-71. 